\section{Plasma-Electron Oscillations}

DURING the past ten years, a series of investigations on the generation of high-frequency oscillations in thermionic tubes containing gas at low pressure has been made in this laboratory. The vacuum electromagnetic wave-lengths involved, usually well below $100 \mathrm{~cm}$., and the conditions under which they appear, are in the main consistent with the view that they are plasma-electron oscillations with frequency approximately equal to $10^{4} n^{1 / 2}$ ( $n$ is electron con.

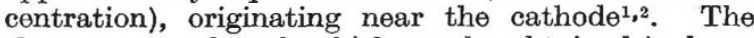
shortest wave-length which can be obtained is, however, not about $15 \mathrm{~cm}$., as indicated by previous publications, but $5 \mathrm{~cm}$. or less. Whatever its exact value, this is likely to be determined by the damping of the oscillatory motion of the electrons by collisions with ions when the ionization is very dense, or by collisions between electrons and gas-molecules at the gas-pressures necessary to produce high electron concentrations readily.

The amplitude and wave-length of the oscillations which can be transmitted to an external circuit depend markedly on position on the current-voltage characteristic of the generating tube. With an oxidecoated cathode, oscillations can usually be detected over wide sections of the characteristic. With a bare filament cathode, the oscillation regions are much more restricted. Often there will be one oscillation region in the infra-saturation section, one at about the saturation ' $k n e \theta$ ', and a third in the saturation section some volts above the 'knee'. No tube which has been studied containing gas at less than $0.01 \mathrm{~mm}$. mercury pressure failed to generate oscillations over some part of its characteristic curve.

The amplitude of the oscillations which can be detected by an internal probe depends considerably on the position of the probe, but the wave-length of the oscillations does not usually change much as the probe is moved. A number of amplitude-distance patterns like those reported by Merrill and $\mathrm{Webb}^{3}$ have been obtained; these, taken in conjunction with the fact that on a number of occasions when a discrete beam of primary electrons could be observed, oscillations could be picked up by the probe only when the beam struck the probe, provide evidence that at least one of the processes operative is velocity modulation of the primary beam in a comparatively small volume of oscillating plasma. From the variation of frequency along the saturation part of the characteristic, it seems that the position of the oscillating volume near the cathode is not constant. There is also evidence that two volumes of plasma with different electron concentrations may oscillate simultaneously and independently, from the fact that a probe will occasionally pick up different frequencies in different parts of a tube. This is in agreement with the theory of plasma electron oscillations, according to which the group velocity of the oscillations is almost zero ${ }^{2}$.

The amplitude of the oscillations which can be obtained externally depends a great deal on electrode geometry, and upon the electrode to which the external circuit is connected. Thus with a simple coaxial electrode system with open ends, by far the strongest oscillations can be set up in a coaxial resonator if the central rod of the latter is joined end-on to the negative end of the filament, and if the filament is held taut and projects a little beyond the end of the anode. There is evidence that effects of this kind are connected in some cases with the movement of the electrons in the magnetic field of the filament-heating current, as the response of the resonator is reduced greatly by eliminating this. Further evidence for the existence of magnetic control is that an asymmetrical tube which normally gives feeble oscillations can be caused to give strong oscillations by bringing a bar magnet near it.

The way in which the plasma-electron oscillations are maintained is still not clear. One obvious possibility is that this is due to interaction between the primary electron beam and the plasma electrons, which depends on their relative speed, but the effects of magnetic fields suggest that some process which occurs with magnetrons may also come into play. It is also possible to draw an analogy between gasfilled diodes and 'hard' triodes giving Barkhausen Kurz oscillations, with a potential maximum in the plasma of the gas taking the part of a positive grid in a 'hard' tube; it is, in fact, known from optical observations and probe measurements in some gasfilled diodes that a region of plasma with unusual properties extends outside the space-charge sheath on the filament for several times the diameter of the latter. Another possibility, suggested by Prof. K. G. Emeléus, is that plasma-electron oscillations outside the filament space-charge sheath are coupled with oscillations inside the sheath in which the potential minimum near the cathode forms and disappears discontinuously. With the complex conditions existing inside even the tubes of simple construction which have been studied, it would not be surprising if more than one of these effects, or others not considered here, should have to be taken into account ultimately.

The work described above has been carried out on behalf of the Director of Physical Research, Admiralty, and I wish to express my thanks to the Admiralty for permission to communicate these results.

\section{E. B. Armstrong}

Department of Experimental Physics,

\section{Queen's University,}

Belfast.

July 24.

1 Penning, Physica, 6, 241 (1926).

${ }^{2}$ Langmuir and Tonks, Phys. Rev., 33, 195 (1929).

${ }^{3}$ Merrill and Webb, Phys. Rev., 55, 1191 (1939).

${ }^{4}$ Druyvesteyn and Warmoltz, Physica, 4, 51 (1937).

\section{Angle of Incidence of the Sun's Rays}

THE radiant heat from the sun received by each square foot of ground affects a number of questions, such as evaporation from the soil, the growth of close-growing crops, and snow conditions. One of the factors involved is (the sine of) the angle of incidence of the sun's rays ; and calculation of this angle, according to aspect and inclination of hillside, and sun's bearing and altitude, may necessitate the solution of many spherical triangles; so the following short cut may be worth noting.

The angle of incidence on a slope at $S$ is equal to the angle of incidence on the parallel plane touching the earth at $T$, the nearer of the two possible tangent points. As this parallel plane corresponds to the level surface at $T$, the sun's altitude at $T$ is the angle required; and if the position of $T$ can be found, that angle can be read directly from altitude (-azimuth) tables, without worrying about spherical triangles, or even azimuth.

But the problem is to find $T$. It lies on the great circle through $S$, the course of which at $S$ corresponds 